\title{
Les éclosions dans l'optique de la syndémie : de nouvelles notions pour améliorer la santé des Autochtones
}

\author{
A Andermann ${ }^{1,2,3 *}$
}

\section{Résumé}

Les approches classiques en matière de prévention et de contrôle des maladies transmissibles en milieux autochtones pourraient tirer profit des nouvelles notions découlant de l'intérêt croissant pour la syndémie. La " syndémie » est un terme utilisé pour décrire un cadre conceptuel visant à comprendre les maladies ou problèmes de santé et la façon dont ils sont exacerbés par le milieu social, économique, environnemental et politique dans lequel vit une population. Encore aujourd'hui, les interventions dans le domaine des maladies transmissibles reposent sur des approches classiques en matière de prévention et de contrôle des éclosions; or, ces stratégies ne sont pas toujours fructueuses à elles seules, en particulier auprès des populations marginalisées et défavorisées. II faut privilégier une approche plus large qui tient compte des facteurs systémiques en cause, qui comprend comment les divers systèmes et politiques contribuent ou nuisent à l'efficacité des interventions et qui détermine les changements structurels nécessaires pour créer des milieux plus favorables et accroître la résilience de la population. Dans le contexte autochtone, que l'accent soit mis sur l'hépatite C, la tuberculose, le VIH ou les maladies d'origine hydrique, il est important de reconnaître a) que les déterminants sociaux contribuent à l'émergence et à la persistance des éclosions, b) que les approches classiques en matière de contrôle des maladies transmissibles sont nécessaires, mais ne suffisent pas à elles seules et c) que l'utilisation d'une approche " syndémique » pourrait permettre de tirer profit d'une action à plusieurs niveaux visant à s'attaquer aux causes profondes du mauvais état de santé et à favoriser l'élaboration de stratégies plus efficaces pour améliorer la santé des Autochtones et réduire les inégalités en matière de santé.

\author{
Affiliations \\ ${ }^{1}$ Direction générale de la santé \\ des Premières nations et des \\ Inuits, Santé Canada, Montréal \\ (Québec) \\ ${ }^{2}$ Département de médecine \\ familiale, Département \\ d'épidémiologie, de \\ biostatistique et de santé au \\ travail, Université McGill, Montréal \\ (Québec) \\ ${ }^{3}$ Département de santé publique, \\ Conseil Cri de la santé et des \\ services sociaux de la Baie James, \\ Montréal (Québec)
}

Correspondance : anne. andermann@mail.mcgill.ca

Citation proposée : Andermann A. Les éclosions dans l'optique de la syndémie : de nouvelles notions pour améliorer la santé des Autochtones. Relevé des maladies transmissibles au Canada. 2017;43(6):140-8.

https://doi.org/10.14745/ccdr.v43i06a02f

\section{Introduction}

Un consensus de plus en plus large se dégage au Canada sur la nécessité d'améliorer l'état de santé des Autochtones. Il est bien connu que les Autochtones ont, en moyenne, une espérance de vie plus courte et un fardeau de maladies chroniques plus lourd que le reste de la population canadienne. Les maladies transmissibles touchent également les Autochtones du Canada de façon disproportionnée. À titre d'exemple, la population autochtone représente environ $4,3 \%$ de la population totale du Canada, mais elle supporte $17 \%$ du fardeau de la tuberculose. Autre disparité, les nouvelles infections sont 270 fois plus susceptibles de se manifester chez des Inuits que chez des non-Autochtones nés au Canada (1). Plusieurs facteurs contribueraient à ces écarts, notamment des facteurs historiques, les conditions de vie dans les logements surpeuplés et les défis liés au système de santé. De plus, la coexistence de problèmes de santé tels que le $\mathrm{VIH}$, le diabète et la consommation d'alcool et de drogues augmente le risque qu'une infection tuberculeuse latente évolue en tuberculose active (2).

Il existe depuis peu un intérêt croissant pour la notion de syndémie au sein de la communauté mondiale de la santé publique. La "syndémie » est un terme utilisé pour décrire un cadre conceptuel visant à comprendre les maladies ou problèmes de santé et la façon dont ils sont exacerbés par le milieu social, économique, environnemental et politique dans lequel vit une population. Cette notion décrit la manière dont des problèmes de santé coexistants peuvent s'exacerber les uns les autres et ainsi accroître la vulnérabilité des populations marginalisées et défavorisées et aggraver les effets sur leur santé (3). Il serait certainement bénéfique de repenser les stratégies actuelles de prévention et de contrôle des maladies transmissibles dans l'optique de la " syndémie », afin d'élaborer des interventions qui vont au-delà des approches classiques (c.-à-d. la vaccination et le suivi des contacts), de manière à inclure des politiques et des programmes qui s'attaquent aux causes profondes sous-jacentes, et souvent structurelles.

La notion de syndémie est étroitement liée à l'équité en matière de santé et aux déterminants sociaux de la santé (4). L'Organisation mondiale de la Santé définit les déterminants sociaux comme « les circonstances dans lesquelles les individus naissent, grandissent, vivent, travaillent et vieillissent ainsi que les systèmes mis en place pour faire face à la maladie » (5), comme les politiques économiques, les normes sociales et les régimes politiques. Les personnes qui vivent dans des conditions défavorables sont souvent davantage exposées aux facteurs 
externes qui menacent leur santé et sont moins en mesure de s'en protéger, ce qui se traduit souvent par de pires résultats sur la santé. À titre d'exemple, selon une étude multi-cohortes et une méta-analyse récemment publiées dans The Lancet, et portant sur plus de 1,7 million de patients suivis durant une période moyenne de 13 ans, les participants âgés de 40 à 85 ans de statut socioéconomique inférieur présentaient un taux de mortalité prématurée d'environ $40 \%$ plus élevé et mouraient en moyenne deux ans plus tôt que les participants d'un statut socioéconomique plus élevé (6).

Les déterminants sociaux de la santé incluent un large éventail de facteurs, dont le revenu, le soutien social, le développement durant la petite enfance, I'éducation, l'emploi, le logement et le sexe (7). Dans le contexte autochtone, en plus de ces facteurs explicatifs sociaux, d'autres facteurs encore plus profondément ancrés, comme les antécédents de colonisation, l'ambiguïté quant au partage des compétences, le racisme structurel, les traumatismes intergénérationnels et même le droit à la propriété foncière et l'émancipation, peuvent tous exercer une grande influence sur la santé de la population, et ce, d'une façon souvent insidieuse (8). De fait, il existe de nombreux cadres qui tentent d'expliquer ce que sont les déterminants sociaux, comment ces facteurs interagissent et influent sur la santé et les moyens à prendre pour réduire les inégalités en matière de santé (9). En ce qui a trait à la santé des Autochtones, l'un des cadres les plus souvent utilisés est le Modèle holistique de planification et de politique des Premières nations élaboré par l'Assemblée des Premières nations (10). Ce modèle souligne l'importance de l'autonomie gouvernementale et de la continuité culturelle, deux éléments que Chandler et Lalonde ont décrits comme étant les fondements de la résilience et du bien-être (11). Une collectivité forte est essentielle au maintien d'une bonne santé, et les rapports (au sein d'une collectivité, entre les collectivités ainsi qu'avec les institutions officielles) sont essentiels au renforcement du capital social (12). Dans ce modèle, la roue médicinale exerce une grande influence holistique (c.-à-d. accent sur les aspects physique, mental, émotionnel et spirituel de la santé), tout comme la prise en compte de la santé durant toutes les étapes de la vie (c.-à-d. chez les enfants, les jeunes, les adultes et les aînés).

Bien que l'on puisse admettre intuitivement que des facteurs plus vastes entrent en jeu, on peut se demander concrètement comment cette nouvelle approche syndémique pourrait contribuer à réduire la morbidité et la mortalité associées aux maladies transmissibles. Le présent article a pour but d'explorer comment l'application d'une approche syndémique peut aider à améliorer la prévention et le contrôle des maladies infectieuses chez les populations autochtones du Canada.

\section{Analyse}

L'utilité de l'approche syndémique pour renforcer la prévention et le contrôle des maladies infectieuses en milieux autochtones repose sur trois hypothèses fondamentales : les déterminants sociaux de la santé contribuent largement à l'émergence et à la persistance des éclosions; un certain nombre de menaces que posent les maladies infectieuses ne pourront être maîtrisées de façon optimale qu'en tenant compte des déterminants sociaux de la santé, et l'utilisation d'une approche syndémique peut favoriser l'élaboration de nouvelles approches en matière de soins cliniques, de santé de la population et de politique publique visant à promouvoir la santé de la population autochtone et à réduire les inégalités.

\section{Les déterminants sociaux qui contribuent aux éclosions}

Il est de plus en plus admis que (traduction) « la pauvreté, le surpeuplement, le déplacement des populations, les systèmes de santé déficients, l'accès inadéquat à de l'eau salubre et à des mesures d'hygiène et l'état de santé de populations précises sont tous des facteurs qui contribuent aux épidémies et à l'éclosion de maladies émergentes " (13). Les personnes qui vivent dans des milieux physiques et sociaux dégradés sont plus à risque de contracter des maladies transmissibles, de les propager et même d'en mourir (14).

Ce constat vaut également pour les populations autochtones, chez qui il a été démontré que « vivre dans un logement qui a besoin de réparations majeures; ne pas avoir terminé ses études secondaires; être sans emploi... vivre en situation d'insécurité alimentaire (au cours des 12 mois précédents et) n'avoir personne sur qui compter en période de besoin " sont associés à des résultats négatifs en matière de santé (15). Or, il est bien documenté que les conditions de vie de bon nombre d'Autochtones au Canada sont beaucoup plus précaires que celles de leurs homologues non autochtones. En 2011, au Canada, « $28 \%$ des Premières Nations vivant dans une réserve et $30 \%$ des Inuits habitaient dans un logement surpeuplé ", comparativement à $4 \%$ des Canadiens non autochtones (16). De même, parmi les adultes autochtones âgés de 25 à 64 ans, " 28,9 \% n'avaient aucun certificat, diplôme ou grade ", alors que la proportion correspondante chez les non-Autochtones était de $12,1 \%$ (17). Hors réserve, " environ un ménage (autochtone) sur cinq $(20,9 \%)$ était en situation d'insécurité alimentaire, dont 8,4 $\%$ en situation d'insécurité alimentaire grave ", ce qui est trois fois plus élevé que les proportions observées chez les ménages non autochtones, dont 7,2 \% étaient en situation d'insécurité alimentaire et $2,5 \%$ en situation d'insécurité alimentaire grave (18).

Il existe une corrélation manifeste entre ces conditions de vie et les taux plus élevés de maladies transmissibles, notamment de tuberculose, de Staphylococcus aureus résistant à la méthicilline (SARM) acquis dans la communauté et d'infections transmissibles sexuellement et par le sang (ITSS) chez les Autochtones que chez les non-Autochtones du Canada (19). De fait, une documentation abondante établit un lien entre les taux plus élevés de $\mathrm{VIH}$ chez les populations autochtones et des facteurs tels que la violence, la stigmatisation et la discrimination, combinés à la méfiance envers le système de santé, (traduction) « ce qui contribue à la situation défavorable vis-à-vis du VIH et aux mauvais résultats en matière de santé observés chez les peuples autochtones » (19). La récente éclosion de $\mathrm{VIH}$ chez les peuples autochtones de la Saskatchewan, où les taux d'incidence sont 11 fois supérieurs à la moyenne nationale, témoigne bien de ce fait (20). L'élaboration $d$ 'interventions et de modèles de soins efficaces exige l'adoption d'approches à facettes multiples qui permettent de s'attaquer aux divers aspects en cause (21), notamment la prise en compte des expériences de vie traumatiques (22), y compris la violence sexuelle (23), ainsi que la prise en charge des comorbidités, y compris les autres infections transmissibles sexuellement comme la chlamydia qui, selon les estimations, " est près de sept fois plus fréquente chez les adultes des Premières Nations que dans la population générale " (24). II serait possible de créer des approches adaptées à la réalité culturelle pour lutter contre 
les éclosions de $\mathrm{VIH}$ dans les populations autochtones, grâce auxquelles $90 \%$ des personnes vivant avec le VIH connaîtraient leur état sérologique, seraient traitées et obtiendraient une suppression virale. Selon un chef autochtone, (traduction) «la communication et la confidentialité sont la clé » (25).

Cependant, un rapport récent intitulé First Peoples, Second Class Treatment décrit les obstacles structurels qui nuisent à l'accès à des soins de qualité et avec lesquels bon nombre de peuples autochtones doivent encore composer sur une base quotidienne (26). D'autres obstacles structurels nuisent à la satisfaction d'un éventail de besoins fondamentaux tels que l'accès à la nourriture, à un logement de qualité et à l'eau potable. La difficulté chronique d'avoir accès à de l'eau potable dans les réserves, et l'attention que cette question a suscitée dans les médias (27), ont amené certaines personnes à utiliser d'autres sources d'eau non salubres, ce qui a causé l'apparition de maladies d'origine hydrique (28). Cette situation complexe causée par la multitude d'épidémies coexistantes représente une véritable syndémie où « les effets cumulatifs de multiples facteurs engendrant un désavantage social accentuent les résultats négatifs dans la vie » (15). En plus des programmes verticaux (c.-à-d. approches axées sur une maladie unique pour la prévention et le contrôle de maladies transmissibles), il serait utile de privilégier davantage les approches " axées sur la personne » et fondées sur des systèmes pour s'attaquer à cet ensemble étroitement lié de problèmes de santé et problèmes sociaux.

\section{Les approches classiques sont nécessaires mais insuffisantes à elles seules}

Il ne fait aucun doute que les approches classiques d'intervention en cas d'éclosion ( $p$. ex. le suivi des contacts et le traitement des cas infectieux) et la promotion des pratiques de prévention (p. ex. I'hygiène des mains, la vaccination et les pratiques sexuelles à risques réduits) demeurent le fondement des mesures de prévention et de contrôle des maladies transmissibles (29). Cependant, pour réduire vraiment l'incidence des maladies et la mortalité qui y est associée, il faut recourir à d'autres outils pour mieux comprendre non seulement les facteurs du système de santé, mais aussi les systèmes sociaux, économiques et politiques plus vastes qui sont en place, et pour mieux y répondre (30). II est depuis longtemps reconnu que la clé du succès de la prévention des éclosions et des interventions en cas d'éclosion est de (traduction) « travailler avec diligence, de tisser des liens avec des collègues et $d$ 'assurer une communication efficace des idées et de l'information entre les différents niveaux de compétence et diverses disciplines scientifiques » (29).

En raison de l'augmentation des voyages internationaux et de l'interconnectivité de notre société mondiale, il devient de plus en plus important d'adopter des approches plus larges qui tiennent compte des préoccupations géopolitiques et de l'évolution des programmes politiques pour assurer la sécurité sanitaire à l'échelle nationale et mondiale (31). II a été soutenu que, malgré la révision en 2005 du Règlement sanitaire international, la prévention des urgences de santé publique de préoccupation internationale (p. ex. les virus Ebola et Zika) nécessitera un rehaussement des investissements dans les systèmes de santé, en particulier dans les pays à revenu faible ou intermédiaire (32). Au cours des derniers mois, les Nations Unies ont fait de l'augmentation de la résistance aux antimicrobiens I'une de leurs priorités, soulignant ainsi la nécessité d'adopter une approche mondiale unifiée pour réduire les menaces que présentent les maladies transmissibles pour la santé (33). Il est de plus en plus manifeste que la prévention ne peut pas viser uniquement l'individu, par des messages tels que "Lavez-vous les mains ", "Portez le condom » et "Évitez de partager des aiguilles ", car la modification des comportements individuels n'est qu'une des pièces d'un casse-tête beaucoup plus grand.

En fait, les occasions manquées en matière de prévention et l'accès inéquitable aux soins de santé ont été cités comme d'importants facteurs expliquant l'inefficacité du système de santé (34), ce qui mène à des résultats négatifs sur la santé. Les dirigeants des systèmes de soins de santé sont de plus en plus conscients de la nécessité d'adopter une approche axée sur les déterminants sociaux et sur la santé de la population, non seulement pour améliorer la santé, mais aussi pour équilibrer les budgets, notamment en (traduction) « réduisant la demande de soins de santé et en contribuant à la viabilité du système de santé » (35). Le secteur de la santé peut jouer un important rôle de premier plan dans l'élimination des inégalités en matière de santé, en faisant de la prise en compte des déterminants sociaux une priorité du secteur, ainsi qu'en intégrant la réduction des disparités dans les politiques, programmes et services de santé (36).

En ce qui a trait à la santé des Autochtones, les appels à l'action de la Commission de vérité et réconciliation peuvent servir d'importants points de départ et de catalyseurs pour amorcer de tels changements (37). La Commission de vérité et réconciliation propose « d'adopter et de mettre en œuvre la Déclaration des Nations Unies sur les droits des peuples autochtones dans le cadre de la réconciliation ", laquelle stipule que "les peuples autochtones sont égaux à tous les autres peuples, tout en reconnaissant le droit de tous les peuples d'être différents, de s'estimer différents et d'être respectés en tant que tels » (38). Les appels à l'action de la Commission de vérité et réconciliation et la Déclaration des Nations Unies soulignent la nécessité de promouvoir une plus grande autodétermination ainsi qu'une amélioration de la protection de l'enfance, du développement économique, de la justice, de la culture, de la langue et de l'éducation pour les peuples autochtones, tous d'importants déterminants sociaux de la santé. Dans ses appels à l'action, la Commission de vérité et réconciliation demande notamment "d'établir des objectifs quantifiables pour cerner et combler les écarts dans les résultats en matière de santé entre les collectivités autochtones et les collectivités non autochtones (...), dont la mortalité infantile, la santé maternelle, le suicide, la santé mentale, la toxicomanie, l'espérance de vie, les taux de natalité, les problèmes de santé infantile, les maladies chroniques, la fréquence des cas de maladie et de blessure ainsi que la disponibilité de services de santé appropriés ». Puisque le rôle du secteur de la santé est d'améliorer la santé (et pas seulement de créer des services de santé) (39), les mesures axées sur les déterminants sociaux s'inscrivent parfaitement dans ce mandat. De plus, les outils nécessaires à l'atteinte de ces objectifs sont à portée de main si l'on adopte une approche syndémique.

\section{Une approche fondée sur la syndémie pour s'attaquer aux causes fondamentales}

Des stratégies efficaces qui tiennent compte des déterminants sociaux de la santé ont été élaborées au cours des dernières décennies à l'intention des travailleurs de la santé de première ligne qui interviennent dans des milieux cliniques occupés (40), des professionnels de la santé publique travaillant auprès de la population (41) et même des décideurs qui élaborent les 
politiques publiques (42). Dans l'analyse qui suit, nous examinons comment appliquer certaines de ces pratiques exemplaires à une approche syndémique, dans le but d'améliorer la santé globale et de réduire les inégalités chez les populations autochtones.

\section{Pratiques cliniques exemplaires pour l'intégration de la syndémie auprès des peuples autochtones}

Il existe aujourd'hui des pratiques exemplaires bien définies permettant d'agir sur les déterminants sociaux de la santé dans la pratique clinique, que ce soit au niveau des soins aux patients, de la pratique ou de la collectivité (tableau 1). Ces pratiques peuvent servir de compléments utiles aux stratégies classiques visant à prévenir et à gérer les maladies transmissibles chez les populations autochtones.

\section{Tableau 1 : Douze pratiques exemplaires pour intégrer la syndémie à la pratique clinique}

\begin{tabular}{|c|c|}
\hline Approche & Pratiques exemplaires \\
\hline \multirow[t]{5}{*}{$\begin{array}{l}\text { Au niveau } \\
\text { des soins aux } \\
\text { patients }\end{array}$} & $\begin{array}{l}\text { Traiter les patients avec dignité et respect et créer } \\
\text { un environnement sûr pour la communication de } \\
\text { l'information }\end{array}$ \\
\hline & $\begin{array}{l}\text { Questionner les patients sur les problèmes sociaux, } \\
\text { en faisant preuve de sensibilité et d'empathie }\end{array}$ \\
\hline & $\begin{array}{l}\text { Allouer un peu plus de temps à l'examen des besoins } \\
\text { complexes en matière de santé et de services sociaux }\end{array}$ \\
\hline & $\begin{array}{l}\text { Connaître les ressources locales vers qui diriger les } \\
\text { patients }\end{array}$ \\
\hline & $\begin{array}{l}\text { Aiguiller les patients et les aider à avoir accès aux } \\
\text { prestations et services de soutien disponibles }\end{array}$ \\
\hline \multirow{3}{*}{$\begin{array}{l}\text { Au niveau de } \\
\text { la clinique } \\
\text { ou du poste } \\
\text { de soins } \\
\text { infirmiers }\end{array}$} & $\begin{array}{l}\text { Envisager l'ajout de rappels dans le dossier médical } \\
\text { ou la mise en place de systèmes de rappel pour } \\
\text { repérer les patients à risque }\end{array}$ \\
\hline & $\begin{array}{l}\text { Envisager des approches participatives favorisant la } \\
\text { participation d'autres cliniciens à votre pratique afin } \\
\text { de créer une culture de réflexion et une approche } \\
\text { d'intervention plus « en amont " }\end{array}$ \\
\hline & $\begin{array}{l}\text { Envisager d'autres modèles de soins, comme } \\
\text { l'intervention communautaire ou le recours à des } \\
\text { intervenants-pivots, pour guider les patients dans } \\
\text { l'utilisation des systèmes de santé et services sociaux }\end{array}$ \\
\hline \multirow[t]{4}{*}{$\begin{array}{l}\text { Dans la } \\
\text { collectivité }\end{array}$} & $\begin{array}{l}\text { Communiquer avec des dirigeants locaux pour } \\
\text { discuter des défis sanitaires et sociaux qui sont } \\
\text { fréquents dans la collectivité }\end{array}$ \\
\hline & $\begin{array}{l}\text { Utiliser l'expérience clinique et les données de } \\
\text { recherche pour promouvoir les changements sociaux }\end{array}$ \\
\hline & $\begin{array}{l}\text { Participer à l'évaluation des besoins de la collectivité } \\
\text { et à la planification des services de santé }\end{array}$ \\
\hline & $\begin{array}{l}\text { Établir des partenariats avec des groupes } \\
\text { communautaires, des dirigeants de la santé } \\
\text { publique et des dirigeants locaux pour promouvoir } \\
\text { des initiatives de collaboration visant à réduire les } \\
\text { inégalités en matière de santé ou à en atténuer les } \\
\text { effets }\end{array}$ \\
\hline
\end{tabular}

Par exemple, une infirmière travaillant dans une collectivité autochtone éloignée peut constater que des infections transmissibles sexuellement sont très courantes dans la population locale, en particulier chez les jeunes. Elle note cependant que peu de jeunes se rendent spontanément au poste de soins infirmiers et qu'ils n'y viennent qu'en cas de blessure aiguë ou d'une autre urgence nécessitant des soins immédiats. L'infirmière essaie donc une approche différente pour établir une relation de confiance avec les jeunes de la collectivité, en organisant chaque semaine un " café » et une soirée cinéma. Ces activités deviennent très populaires et, peu à peu, les jeunes commencent à se confier à l'infirmière et à lui faire part de leurs problèmes; l'infirmière est alors en mesure de les conseiller sur des questions telles que de saines relations, la planification familiale, la consommation d'alcool et de drogues et la prévention de la violence. Au fil du temps, plus de jeunes se rendent à la clinique pour recevoir des conseils sur la santé en matière de sexualité et sur le dépistage des maladies infectieuses ou encore des traitements, et ils sont dirigés au besoin vers divers services de santé et services sociaux. Lorsque l'infirmière comprend mieux les principales difficultés et la dynamique du milieu local, elle commence à réfléchir à ce qui pourrait être fait à l'échelle communautaire pour créer des environnements plus positifs en matière de santé; elle entame un dialogue avec le chef du conseil des jeunes, le directeur de l'école, des aînés, des aidants naturels et d'autres membres de la communauté et ces efforts mènent à la tenue d'une réunion sur la santé des jeunes où l'on discute de la voie à suivre en vue d'amorcer de plus grands changements à l'échelle communautaire. Bien qu'il n'existe pas de solutions " universelles », les travailleurs de la santé de première ligne peuvent être d'importants catalyseurs de changements pour agir sur les déterminants sociaux de la santé à plusieurs niveaux.

\section{Approches syndémiques en matière de santé publique et de politiques visant les populations autochtones}

Outre la mise en œuvre des domaines d'action de la Charte d'Ottawa pour la promotion de la santé (43), l'élaboration de politiques en matière de santé publique et la création d'environnements positifs pour la santé, les efforts plus récents visant à réduire les inégalités en matière de santé ont été axés principalement sur l'action intersectorielle et sur l'intégration de "la santé dans toutes les politiques ». L'action intersectorielle requiert la collaboration de multiples secteurs, dont ceux de l'éducation, de la justice et de l'emploi, pour s'attaquer aux problèmes complexes. L'approche de « la santé dans toutes les politiques " requiert la participation et le soutien de tous les ordres de gouvernement, ainsi qu'un solide leadership aux plus hauts niveaux (44). Les exemples de pratiques exemplaires sur la façon de créer des changements structurels sont de plus en plus nombreux, même dans le contexte des maladies transmissibles, qu'il s'agisse d'établir les objectifs en matière de politiques, de renforcer les capacités, de promouvoir les partenariats à plusieurs niveaux entre les secteurs ou de tenir la direction responsable d'amorcer de réels changements (45).

Un élément clé du travail intersectoriel et de l'approche de « la santé dans toutes les politiques " est la participation significative des personnes qui seront les plus touchées par les décisions qui seront prises. Que les décisions visent une population ou une politique, les choix éthiques exigent non seulement que les avantages globaux l'emportent sur les préjudices, et que ces avantages et préjudices soient répartis équitablement (46), mais également que la prise de décisions repose sur un processus équitable et transparent (47). La participation des peuples autochtones à la prise des décisions qui ont une incidence sur leur santé exige la mise en place d'un processus permanent qui permet de (traduction) « créer des occasions pour la collectivité de se faire entendre et d'agir pour modifier les conditions sociales et structurelles reconnues pour exercer un large éventail d'effets sur la santé de la collectivité » (48). Les peuples 
autochtones devraient être consultés sur chaque aspect de la prise de décisions qui a une incidence sur leur vie de tous les jours. Selon la Déclaration des Nations Unies sur les droits des peuples autochtones, "Les peuples autochtones ont le droit de participer à la prise de décisions sur des questions qui peuvent concerner leurs droits, par l'intermédiaire de représentants qu'ils ont eux-mêmes choisis conformément à leurs propres procédures, ainsi que le droit de conserver et de développer leurs propres institutions décisionnelles ॥ (38). II y a un dicton qui dit que, même avec les meilleures intentions du monde, "rien pour nous sans nous ». La participation, I'autonomisation et l'autodétermination dans l'élaboration et la définition de solutions à des problèmes complexes sont en soi d'importants résultats ( $p$. ex. un processus équitable et transparent fait partie intégrante de la solution).

Par comparaison avec le milieu clinique, les décisions qui touchent des populations ou des politiques publiques sont plus complexes et font intervenir un nombre beaucoup plus important d'intervenants (49). Il est particulièrement important que les nombreux partenaires différents travaillent de concert à l'atteinte des objectifs communs, qui sont d'améliorer la santé et de réduire les inégalités, lorsqu'il s'agit de questions de compétence partagée, ce qui est très fréquent lorsque les questions concernent la santé des Autochtones (50). La documentation fournit quelques exemples d'une telle collaboration intersectorielle axée sur l'amélioration de la santé des Autochtones du Canada (51); cette collaboration commence à s'établir mais, avant que l'on observe une véritable réduction des inégalités en matière de santé des Autochtones, elle devra faire partie intégrante du mode opératoire.

Outre les structures tripartites officielles (réunissant des représentants des gouvernements fédéral, provinciaux et autochtones, comme l'autorité sanitaire des Premières nations de la Colombie-Britannique et le Comité intergouvernemental de la santé des Premières nations du Manitoba) (52) qui sont de plus en plus utilisées, il existe de nombreuses façons d'établir des partenariats intersectoriels et pluri-institutionnels pour dissiper les ambiguïtés sur le partage des compétences. À titre d'exemple, la prévention des maladies transmissibles d'origine hydrique dans les réserves est un défi de taille qui exige la collaboration de nombreuses personnes et organisations différentes. Les lois qui régissent l'accès à l'eau potable ne sont pas sans lacunes (53). La crise de Walkerton nous a en effet enseigné que, même dans des milieux non autochtones, des défaillances dans la surveillance de la qualité de l'eau et la communication peuvent mener à l'éclosion de maladies d'origine hydrique et même à des décès, tous potentiellement évitables. L'enquête sur Walkerton a fait ressortir l'importance de procéder régulièrement à des analyses de l'eau, d'en consigner les résultats et de communiquer rapidement les résultats anormaux aux responsables de la santé publique, afin d'assurer la mise en place de mesures préventives appropriées, p. ex. les avis concernant la qualité de l'eau potable et systèmes adéquats de traitement de l'eau (54). Ces mesures s'inscrivent dans une approche à barrières multiples qui vise à protéger les citoyens contre les préjudices et qui s'applique également au contexte autochtone (55).

Il est important que l'élaboration de solution pour protéger les peuples autochtones contre les maladies d'origine hydrique se fasse en présence d'Autochtones et d'autres partenaires réunis autour d'une même table (ou durant une même téléconférence), afin que tous puissent convenir des détails de leur application « sur le terrain ». Il faut réunir le médecin hygiéniste régional et le gestionnaire régional du Programme de santé environnementale de la Direction générale de la santé des Premières nations et des Inuits de Santé Canada, le directeur du logement et de l'infrastructure du bureau régional d'Affaires autochtones et du Nord Canada, l'équipe du ministère provincial de l'Environnement responsable de l'application de la réglementation provinciale sur l'eau, la personne relevant du médecin hygiéniste en chef au ministère provincial de la Santé qui est responsable de la protection de la santé de l'environnement et, bien sûr, des représentants des collectivités autochtones ou des organismes de gouvernance autochtone afin que tous travaillent ensemble dans le cadre d'une première étape nécessaire pour dégager une compréhension commune des problèmes complexes en jeu et proposer des stratégies visant à faciliter la communication et la transparence dans l'atteinte d'objectifs communs.

L'adoption d'une telle approche de collaboration pour résoudre ces problèmes complexes pourrait aider à éviter de créer l'impression qu'il existe " deux poids deux mesures " lorsqu'il s'agit de la gestion de la qualité de l'eau, selon qu'on se trouve sur une réserve ou hors réserve. Si des exemptions sont accordées à certaines municipalités (p. ex. elles ne sont pas tenues d'envoyer chaque mois le nombre requis d'échantillons d'eau aux laboratoires accrédités en raison de leur éloignement et des problèmes de transport), des exemptions comparables pourraient être accordées à des collectivités autochtones qui font face aux mêmes problèmes d'éloignement. La réglementation devrait toutefois être appliquée uniformément, sans égard à l'ethnicité ou aux antécédents culturels des habitants. Si les usines de traitement de l'eau doivent satisfaire à des normes d'agrément précises, ou que les contrôleurs communautaires de la qualité de l'eau doivent posséder une accréditation précise reconnue par les organismes de réglementation, tous devraient travailler à l'atteinte de ces objectifs précis pour s'assurer que tous sont protégés également en vertu de la loi. De cette façon, il sera possible de surmonter les nombreux obstacles organisationnels et structurels qui nuisent aux changements au sein de la population ou des politiques, y compris « le manque de temps ou de ressources, la rigidité des politiques, le manque de soutien de la part de la direction et les cloisonnements ministériels » (56). Une telle solution ne sera peut-être pas rapide; cependant, en allant de l'avant ensemble, il est probable qu'on trouvera des solutions plus efficaces et plus durables aux problèmes complexes et que cela mènera certainement à un processus plus inclusif et plus équitable. En effet, il existe des exemples provenant d'autres provinces et territoires qui illustrent la façon dont la voix des Autochtones a été prise en compte dans les processus de prise de décisions et dont nous pourrions nous inspirer (57).

Comme les changements climatiques marquent le début d'une progression vers le nord des vecteurs de maladies transmissibles, comme les tiques porteuses de la maladie de Lyme au Canada, les connaissances traditionnelles et la sagesse des aînés peuvent être très utiles. Selon un dicton autochtone, « Nous n'héritons pas de la Terre de nos parents, nous l'empruntons à nos enfants (58) ». Ce dicton fait ressortir la sagesse d'une prise de décision qui tient compte des répercussions sur les générations futures. Nous avons beaucoup à apprendre des approches autochtones en matière de santé et de bien-être, non seulement pour notre propre santé, mais aussi pour la santé des animaux et de la Terre, 
et pour nous acquitter de notre responsabilité fiduciaire envers le bien-être de notre planète $(59,60)$.

\section{Conclusion}

Les approches classiques en matière de prévention et de contrôle des maladies transmissibles ne suffisent plus à traiter certaines menaces graves que posent les maladies infectieuses, au Canada et dans le monde entier. Bien que le milieu médical et celui de la santé publique s'intéressent de plus en plus à l'importance d'une approche axée sur les déterminants sociaux, ce n'est que depuis peu que l'on s'intéresse aux inégalités en matière de santé au Canada. Nous avons encore beaucoup à apprendre sur la façon d'évaluer ces inégalités et d'amener les différents ministères et secteurs à mieux collaborer, pour trouver des solutions efficaces aux problèmes de santé et aux problèmes sociaux.

La Commission de vérité et réconciliation souligne la nécessité pour les peuples autochtones de participer davantage à la création de leurs propres systèmes et structures et au façonnement de leur avenir. L'utilisation d'une approche syndémique et la prise en compte des déterminants sociaux pourraient nous aider à mieux comprendre les causes profondes du mauvais état de santé ainsi qu'à mieux agir pour réduire la vulnérabilité et accroître la résilience de tous les Canadiens, y compris les peuples autochtones du Canada. Comme il s'agit d'un nouveau domaine d'action, nous devons mettre en commun nos pratiques exemplaires et nos réussites - tant sur le plan clinique que sur celui de la santé de la population ou des politiques publiques - et en rendre compte, afin de progresser vers la création d'une société en meilleure santé, qui soit plus équitable et plus durable.

\section{Déclaration de l'auteur}

Les points de vue exprimés dans cet article sont ceux de l'auteur et ne reflètent pas nécessairement ceux du gouvernement du Canada.

\section{Conflit d'intérêt}

Aucun.

\section{Remerciements}

Je tiens à remercier le directeur administratif régional, région du Québec, de la Direction générale de la santé des Premières nations et des Inuits de Santé Canada, Richard Budgell, pour son leadership sans prétention dans la mise en œuvre des appels à l'action de la Commission de vérité et réconciliation ainsi que pour ses efforts visant à accroître la sécurisation culturelle et la sensibilisation aux cultures dans son cercle d'influence, sans oublier la $D^{\text {re }}$ Patricia Huston et mes collègues pour leurs suggestions utiles concernant les versions préliminaires de cet article.

\section{Financement}

Mon programme de recherche sur la prise en compte des déterminants sociaux en milieux cliniques (www.mcgill.ca/clear) est appuyé par Grands Défis Canada (GDC), les Instituts de recherche en santé du Canada (IRSC), le Fonds de recherche du Québec - Santé (FRSQ), la Fédération des médecins spécialistes du Québec (FMSQ) et le Centre de recherche St Mary's de Montréal (Canada).

\section{Références}

1. Gallant V, Duvvuri V, McGuire M. La tuberculose — Résumé 2015. Relevé des maladies transmissibles au Canada. 2017;43(3):85-91. Disponible à l'adresse : http://www.phacaspc.gc.ca/publicat/ccdr-rmtc/17vol43/dr-rm43-3-4/ar-04-fra. php.

2. Public Health Agency of Canada. Canadian Tuberculosis Standards, 6th Edition. Ottawa; 2007.

3. Hart L, Horton R. Syndemics: committing to a healthier future. Lancet. 2017;389(10072):888-9. DOI: http://dx.doi. org/10.1016/S0140-6736(17)30599-8. PubMed (https://www. ncbi.nlm.nih.gov/entrez/query.fcgi?cmd=Retrieve\&db=PubM ed\&list_uids=28271826\&dopt=Abstract).

4. Organisation mondiale de la santé. Combler le fossé et une génération; 2008. Disponible à l'adresse : http://www.who. int/social_determinants/thecommission/finalreport/fr/.

5. Organisation mondiale de la santé. Déterminants sociaux de la santé. Geneva; 2008. Disponible à l'adresse : http://www. who.int/social_determinants/fr/ (consulté le 15 mai 2017).

6. Stringhini S, Carmeli C, Jokela M, Avendaño M, Muennig P, Guida F, Ricceri F, d'Errico A, Barros H, Bochud M, Chadeau-Hyam M, Clavel-Chapelon F, Costa G, Delpierre C, Fraga S, Goldberg M, Giles GG, Krugh V, KellyIrving M, Layte R, Lasserre AM, Marmot MG, Preisig M, Shipley MJ, Vollenweider P, Zins M, Kawachi I, Steptoe A, Mackenbach JP, Vineis P, Kivimaki M, . LIFEPATH consortium. Socioeconomic status and the $25 \times 25$ risk factors as determinants of premature mortality: a multicohort study and meta-analysis of 1.7 million men and women. Lancet. 2017;389(10075):1229-37. DOI: http://dx.doi.org/10.1016/ S0140-6736(16)32380-7. PubMed (https://www.ncbi.nlm. nih.gov/entrez/query.fcgi?cmd=Retrieve\&db=PubMed\&li st_uids=28159391\&dopt=Abstract).

7. Les déterminants sociaux de la santé: Les faits. Deuxième édition. Marmot $M$, Wilkinson $R$, eds. Copenhagen: Organisation mondiale de la Santé, Bureau régional de I'Europe, 2003. Disponible à l'adresse : http://www.euro. who.int/fr/publications/abstracts/social-determinants-ofhealth.-the-solid-facts (consulté le 15 mai 2017).

8. Greenwood M, de Leeuw S, Lindsay N, Reading C. Determinants of Indigenous Peoples' Health in Canada; Beyond the Social. Toronto: Canadian Scholars Press, 2017.

9. Conseil canadien des déterminants sociaux de la santé. Examen des cadres relatifs aux déterminants de la Santé. Ottawa; 2015. Disponible à l'adresse : http://nccdh. $\mathrm{ca} / \mathrm{fr} /$ resources/entry/a-review-of-frameworks-on-thedeterminants-of-health. 
10. Assembly of First Nations. First Nations Holistic Policy and Planning a Transitional Discussion Document on the Social Determinants of Health. Ottawa; 2013. Disponible à I'adresse : http://health.afn.ca/uploads/files/sdoh_afn.pdf.

11. Chandler M, Lalonde C. Cultural continuity as a hedge against suicide in Canada's First Nations. Transcult Psychiatry. 1998;35(2):191-219. DOI: http://dx.doi. org/10.1177/136346159803500202.

12. Mignone J, O’Neil J. Social capital as a health determinant in First Nations: an exploratory study in three communities. J Aborig Health. 2005;2(1):26-33.

13. Schiavo R, May Leung M, Brown M. Communicating risk and promoting disease mitigation measures in epidemics and emerging disease settings. Pathog Glob Health. 2014;108(2):76-94. DOI: http://dx.doi.org/10.1179/204 7773214Y.0000000127. PubMed (https://www.ncbi.nlm. nih.gov/entrez/query.fcgi?cmd=Retrieve\&db=PubMed\&li st_uids $=24649867 \&$ dopt $=$ Abstract).

14. Sanders D, Sengupta A, Scott V. Ebola epidemic exposes the pathology of the global economic and political system. Int J Health Serv. 2015;45(4):643-56. DOI: http://dx.doi. org/10.1177/0020731415606554. PubMed (https://www. ncbi.nlm.nih.gov/entrez/query.fcgi?cmd=Retrieve\&db=PubM ed\&list_uids=26391140\&dopt=Abstract).

15. Rotenburg C. Les déterminants sociaux de la santé des membres des Premières Nations de 15 ans et plus vivant hors réserve, 2012. Ottawa; Statistique Canada, 2016. Disponible à l'adresse : http://www.statcan.gc.ca/pub/89653-x/89-653-x2016010-fra.htm.

16. Kelly-Scott K, Smith K. Les peuples autochtones : Feuillet d'information du Canada. Ottawa: Statistique Canada, 2015. Disponible à l'adresse : http://www.statcan.gc.ca/pub/89656-x/89-656-x2015001-eng.pdf (consulté le 15 mai 2017).

17. Statistique Canada. Le niveau de scolarité des peuples autochtones au Canada: Enquête nationale auprès des ménages (ENM), 2011. Ottawa; 2013. Disponible à l'adresse : http://www12.statcan.gc.ca/nhs-enm/2011/assa/99-012-x/99-012-x2011003_3-fra.pdf.

18. Santé Canada. Insécurité alimentaire des ménages au Canada en 2007-2008 : Statistiques et graphiques clés. Ottawa; 2012. Disponible à l'adresse : http://www.hc-sc. gc.ca/fn-an/surveill/nutrition/commun/insecurit/key-statscles-2007-2008-fra.php.

19. Negin J, Aspin C, Gadsden T, Reading C. HIV among Indigenous peoples: A review of the literature on HIV-related behaviour since the beginning of the epidemic. AIDS Behav. 2015 Sep;19(9):1720-34. DOI: http://dx.doi.org/10.1007/ s10461-015-1023-0. PubMed (https://www.ncbi.nlm.nih. gov/entrez/query.fcgi?cmd=Retrieve\&db=PubMed\&lis t_uids=25731659\&dopt=Abstract).

20. Leo G. HIV rates on Sask. reserves higher than some African nations: Saskatchewan on-reserve HIV epidemic 'a real Canadian crisis,' says expert. CBC News; June 3, 2015. Disponible à l'adresse : http://www.cbc.ca/news/canada/ saskatchewan/hiv-rates-on-sask-reserves-higher-than-someafrican-nations-1.3097231.

21. Vogel L. HIV in Saskatchewan merits urgent response. CMAJ. 2015;187(11):793-4. DOI: http://dx.doi.org/10.1503/ cmaj.109-5105. PubMed (https://www.ncbi.nlm.nih. gov/entrez/query.fcgi?cmd=Retrieve\&db=PubMed\&lis t_uids=26124233\&dopt=Abstract).

22. Kirmayer LJ, Gone JP, Moses J. Rethinking historical trauma. Transcult Psychiatry. 2014;51(3):299-319. DOI: http://dx.doi. org/10.1177/1363461514536358. PubMed (https://www. ncbi.nlm.nih.gov/entrez/query.fcgi?cmd=Retrieve\&db=PubM ed\&list_uids=26124233\&dopt=Abstract).

23. Cedar Project Partnership, Pearce M, Christian W, Patterson K, Norris K, Moniruzzaman A, Craib KJP, Schechter MT, Spittal PM. The Cedar Project: historical trauma, sexual abuse and HIV risk among young Aboriginal people who use injection and non-injection drugs in two Canadian cities. Social Science \& Medicine. 2008;66(11):2185-94.

24. Agence de la santé publique du Canada. Les infections transmissibles - Une menace perpétuelle : Rapport de l'administration en chef de la santé publique sur l'état de la santé publique au Canada, 2013. Ottawa; 2013. Disponible à l'adresse : http://www. phac-aspc.gc.ca/cphorsphcrespcacsp/2013/sti-its-fra.php.

25. Craig M, Piller M. First Nations forum focuses on 'Know Your Status' program success to combat HIV: Global News Toronto, Health. Feb 14, 2017. Disponible à I'adresse : http://globalnews.ca/news/3247047/first-nations-forumfocuses-on-know-your-status-program-success-to-combathiv/.

26. Allan B, Smylie J. First Peoples, Second Class Treatment: The Role of Racism in the Health and Well-being of Indigenous Peoples in Canada. Toronto, ON: Wellesley Institute, 2015.

27. Levasseur J, Marcoux J. Bad water: 'Third World' conditions on First Nations in Canada. CBC News; Oct 14, 2015. Disponible à l'adresse : http://www.cbc.ca/news/canada/ manitoba/bad-water-third-world-conditions-on-first-nationsin-canada-1.3269500.

28. Jung J, Skinner K. Maladies d'origine alimentaire et hydrique dans les populations canadiennes autochtones : examen de la portée. Relevé des maladies transmissibles au Canada. 2017;43(1):7-14. Disponible à l'adresse : http://www.phacaspc.gc.ca/publicat/ccdr-rmtc/17vol43/dr-rm43-1/ar-02-fra. php.

29. Gregg M. Field Epidemiology. 3rd ed. Oxford: Oxford University Press, 2008.

30. Arauz MJ, Ridde V, Hernández LM, Charris Y, Carabali M, Villar LÁ. Developing a social autopsy tool for dengue mortality: a pilot study. PLoS One [Internet]. 2015;10(2):e0117455. DOI: http://dx.doi.org/10.1371/ journal.pone.0117455. PubMed (https://www.ncbi.nlm. nih.gov/entrez/query.fcgi?cmd=Retrieve\&db=PubMed\&li st_uids=25658485\&dopt=Abstract).

31. Quinn SC, Kumar S. Health inequalities and infectious disease epidemics: a challenge for global health security. Biosecur Bioterror. 2014;12(5):263-73. DOI: http://dx.doi. org/10.1089/bsp.2014.0032. PubMed (https://www.ncbi.nlm. nih.gov/entrez/query.fcgi?cmd=Retrieve\&db=PubMed\&li st_uids=25254915\&dopt=Abstract).

32. Bennett B, Carney T. Public health emergencies of international concern: global, regional, and local responses 
to risk. Med Law Rev. Epub Mar 31, 2017. PubMed (https:// www.ncbi.nlm.nih.gov/pubmed/28379440?dopt=Abstract).

33. Friedrich MJ. UN Leaders Commit to fight antimicrobial resistance. JAMA. 2016;316(19):1956. PubMed (https://www. ncbi.nlm.nih.gov/pubmed/27838729?dopt=Abstract).

34. Institut canadien d'information sur la santé. Mesurer l'efficacité du système de santé canadien et ses déterminants. Ottawa; 2014. Disponible à l'adresse : https:// www.cihi.ca/sites/default/files/hse_technicalreport_fr_web_0. pdf (consulté le 15 mai 2017).

35. Cohen D, Huynh T, Sebold A, Harvey J, Neudorf C, Brown A. The population health approach: A qualitative study of conceptual and operational definitions for leaders in Canadian healthcare. SAGE Open Med [Internet]. 2014;2:111. DOI: http://dx.doi.org/10.1177/2050312114522618. PubMed (https://www.ncbi.nlm.nih.gov/entrez/ query.fcgi?cmd=Retrieve\&db=PubMed\&list_ uids $=26770704 \&$ dopt $=$ Abstract $)$.

36. Gouvernement du Canada. Groupe de travail sur les disparités en matière de santé du Comité consultatif fédérale-provincial-territorial sur la santé de la population et la sécurité de la santé. Réduire les disparités sur le plan de la santé - Rôle du secteur de la santé : Document de travail. Ottawa; 2004. Disponible à l'adresse : http://www.phac-aspc. gc.ca/ph-sp/disparities/pdf06/disparities_discussion_paper_f. pdf (consulté le 15 mai 2017).

37. Truth and Reconciliation Commission. Truth and Reconciliation of Canada: Calls to Action. Winnipeg; 2015. Disponible à l'adresse : http://www.trc.ca/websites/ trcinstitution/File/2015/Findings/Calls_to_Action_English2. pdf (consulté le 15 mai 2017).

38. Nations Unies. Déclaration des Nations Unies sur les droits des peuples autochtones; 2008. Disponible à l'adresse : http://www.un.org/esa/socdev/unpfii/documents/DRIPS_ fr.pdf (consulté le 15 mai 2017).

39. Asch DA, Volpp KG. What business are we in? The emergence of health as the business of health care. $\mathrm{N}$ Engl J Med. 2012;367(10):888-9. DOI: http://dx.doi.org/10.1056/ NEJMp1206862. PubMed (https://www.ncbi.nlm.nih. gov/entrez/query.fcgi?cmd=Retrieve \&db=PubMed\&lis t_uids=22931228\&dopt=Abstract).

40. Andermann A. Taking action on the social determinants of health in clinical practice; a framework for health professionals. CMAJ [Internet]. 2016;188(17-18):E474-E483. (consulté le April 12, 2017). DOI: http://dx.doi.org/10.1503/ cmaj.160177. PubMed (https://www.ncbi.nlm.nih.gov/ entrez/query.fcgi?cmd=Retrieve\&db=PubMed\&list_ uids $=27503870 \&$ dopt $=$ Abstract).

41. Pauly B, MacDonald M, O'Brian W, Hancock T, Perkin K, Martin W, Riishede J. Health Equity Tools. Victoria, BC: Equity Lens in Public Health, 2013. Disponible à l'adresse : http://nccdh.ca/resources/entry/health-equity-tools (consulté le 15 mai 2017).

42. Welch VA, Petticrew M, O’Neill J, Waters E, Armstrong $R$, Bhutta ZA, Francis D, Koehlmoos TP, Kristjansson E, Pantoja T, Tugwell P. Health equity: evidence synthesis and knowledge translation methods. Syst Rev [Internet]. 2013;2:43. DOl: http://dx.doi.org/10.1186/2046-
4053-2-43. PubMed (https://www.ncbi.nlm.nih.gov/ pubmed/28379440?dopt=Abstract).

43. Organisation mondiale de la Santé. Ottawa Charter for Health Promotion. Geneva; 1986. Disponible à l'adresse : http://www.who.int/healthpromotion/conferences/previous/ ottawa/en/ (consulté le 15 mai 2017).

44. de Leeuw E. Engagement of sectors other than health in integrated health governance, policy, and action. Annu Rev Public Health. 2017;38:329-49. DOI: http:// dx.doi.org/10.1146/annurev-publhealth-031816-044309. PubMed (https://www.ncbi.nlm.nih.gov/ pubmed/28125390?dopt=Abstract).

45. Dean HD, Williams KM, Fenton KA. From theory to action: applying social determinants of health to public health practice. Public Health Reports. 2013;128(Suppl 3):1-4. DOI: http://dx.doi.org/10.1177/00333549131286S301. PubMed (https://www.ncbi.nlm.nih.gov/pmc/articles/PMC3945442/).

46. Kass NE. An ethics framework for public health. Am J Public Health [Internet]. 2001;91(11):1776-82. DOI: http://dx.doi. org/10.2105/AJPH.91.11.1776. PubMed (https://www.ncbi. nlm.nih.gov/pubmed/11684600?dopt=Abstract).

47. Gruskin S, Daniels N. Process is the point. Justice and human rights: priority setting and fair deliberative process. Am J Public Health [Internet]. 2008; 98(9): 1573-7. DOI: http:// dx.doi.org/10.2105/AJPH.2007.123182. PubMed (https:// www.ncbi.nlm.nih.gov/entrez/query.fcgi?cmd=Retrieve $\& d b=$ PubMed\&list_uids=18633088\&dopt=Abstract).

48. Sabo S, Ingram M, Reinschmidt KM, Schachter K, Jacobs L, Guernsey de Zapien J, Robinson L, Carvajal S. Predictors and a framework for fostering community advocacy as a community health worker core function to eliminate health disparities. Am J Public Health [Internet]. 2013;103(7):e67-73. DOI: http://dx.doi.org/10.2105/AJPH.2012.301108. PubMed (https://www.ncbi.nlm.nih.gov/entrez/query.fcgi?cmd=Retrie ve\&db=PubMed\&list_uids=23678904\&dopt=Abstract).

49. Andermann A. Evidence for Health: From Patient Choice to Global Policy. Cambridge: Cambridge University Press, 2013. Disponible à l'adresse : http://www.cambridge.org/gb/ knowledge/isbn/item6945171/ (consulté le 15 mai 2017).

50. National Collaborating Centre for Aboriginal Health. Aboriginal health: a patchwork of policies and legislation. Victoria; 2011. Disponible à l'adresse : http://www.nccahccnsa.ca/252/Aboriginal_health_A_patchwork_of_policies_ and_legislation.nccah\#1 (consulté le 15 mai 2017).

51. Lavoie JG. Policy silences: why Canada needs a national First Nations, Inuit and Métis health policy. Int J Circumpolar Health. 2013;72(1):22690-8. DOI: http://dx.doi.org/10.3402/ ijch.v72i0.22690. PubMed (https://www.ncbi.nlm.nih. gov/entrez/query.fcgi?cmd=Retrieve\&db=PubMed\&lis t_uids=24380077\&dopt=Abstract).

52. Kolahdooz F, Nader F, Yi K, Sharma S. Understanding the social determinants of health among Indigenous Canadians: priorities for health promotion policies and actions. Glob Health Action [Internet]. 2015;8(1):27968. DOI: http://dx.doi. org/10.3402/gha.v8.27968. PubMed (https://www.ncbi.nlm. nih.gov/entrez/query.fcgi?cmd=Retrieve\&db=PubMed\&li st_uids=28156855\&dopt=Abstract). 
53. Pardy B. Seven deadly sins of Canadian water law. J Env Law \& Prac. 2004;13:89-110.

54. O'Connor DR. Report of the Walkerton Inquiry: The Events of May 2000 and Related Issues. Toronto: Queen's Printer for Ontario, 2002. Disponible à l'adresse : http://www.archives. gov.on.ca/en/e_records/walkerton/report1/ (consulté le 15 mai 2017).

55. Le Conseil Canadien des ministres de l'environnement. Produit conjoinement par le Comité fédéral-provincialterritorial sur l'eau potable et par le Groupe de de travail sur la qualité de l'eau du CCME. De la source au robinet: Guide d'application de l'approche à barrières multiples pour une eau potable saine. Ottawa; 2004. Disponible à l'adresse : http://www.ccme.ca/files/Resources/fr_water/fr_source_to_ tap/mba_guidance_doc_f.pdf (consulté le 15 mai 2017).

56. Rideout K, Oickle D. Equity in Environmental Health Practice: Findings of a Pilot Study. Vancouver, BC: The National Collaborating Centre for Environmental Health (NCCEH) and the National Collaborating Centre for Determinants of Health (NCCDH), 2015. Disponible à l'adresse : http://www.ncceh. ca/sites/default/files/Equity_EH_practice_April_2015.pdf (consulté le 15 mai 2017).
57. Lavoie JG, Boulton FA, Gervais L. Regionalization as an opportunity for meaningful Indigenous participation in healthcare: Comparing Canada and New Zealand. The International Indigenous Policy Journal [Internet]. 2012;3(1). Disponible à l'adresse : http://ir.lib.uwo.ca/iipj/vol3/iss1/2/ (consulté le 12 avril 2017). DOI: http://dx.doi.org/10.18584/ iipj.2012.3.1.2.

58. Barry J. Rethinking Green Politics: Nature, Virtue and Progress. London: SAGE Publishing, 1999.

59. Griffin PM, Karmali MA. Emerging public health challenges of shiga toxin-producing Escherichia coli related to changes in the pathogen, the population, and the environment. Clin Infect Dis. 2017;64(3):371-6. DOI: http://dx.doi. org/10.1093/cid/ciw708. PubMed (https://www.ncbi.nlm. nih.gov/entrez/query.fcgi?cmd=Retrieve\&db=PubMed\&li st_uids=27986670\&dopt=Abstract).

60. Ruscio BA, Brubaker M, Glasser J, Hueston W, Hennessy T. One Health - a strategy for resilience in a changing arctic. Int J Circumpolar Health. 2015;74(1):27913. DOI: http://dx.doi. org/10.3402/ijch.v74.27913. PubMed (https://www.ncbi.nlm. nih.gov/entrez/query.fcgi?cmd=Retrieve\&db=PubMed\&li st_uids $=28417805 \&$ dopt $=$ Abstract).

\section{Deux publications des \\ Lignes directrices canadiennes sur les infections transmissibles sexuellement (LDCITS)}
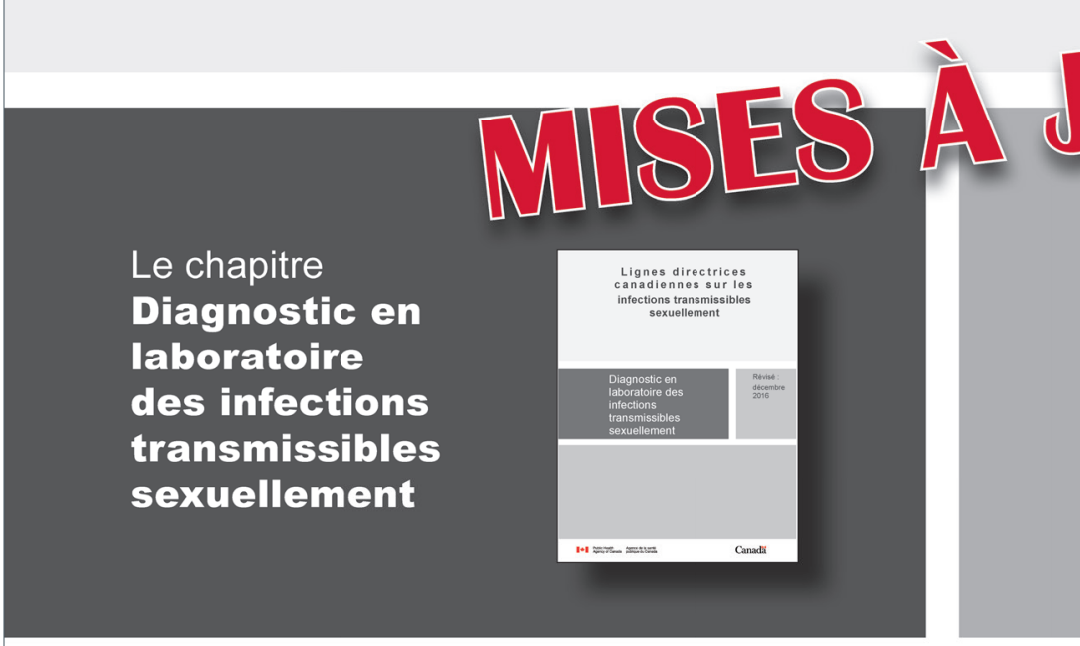

Le chapitre

Diagnostic en

laboratoire

des infections

transmissibles

sexuellement
Joun
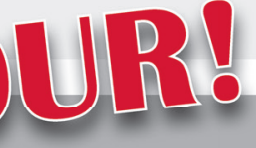

Sommaire des mises à jour : 2016 pour les changementsclés du contenu des LDCITS

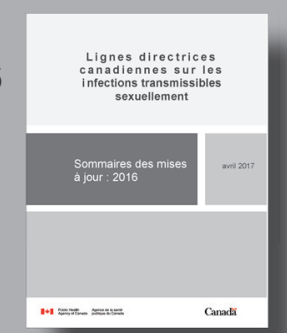

Assurez-vous d'utiliser les recommandations les plus récentes Les publications sont GRATUITES. Visitez Canada.ca et recherchez "LDCITS ». 